“C 2016 IEEE. Personal use of this material is permitted. Permission from IEEE must be obtained for all other uses, in any current or future media, including reprinting/republishing this material for advertising or promotional purposes, creating new collective works, for resale or redistribution to servers or lists, or reuse of any copyrighted component of this work in other works." 


\title{
An Adaptive-then-Combine Dynamic State Estimation Considering Renewable Generations in Smart Grids
}

\author{
Md Masud Rana, Li Li and Steven W. Su \\ Faculty of Engineering and Information Technology \\ University of Technology Sydney, Broadway, NSW 2007, Australia
}

\begin{abstract}
The penetration of renewable distributed energy resources such as wind turbine has been dramatically increased in distribution networks. Due to the intermittent property, the wind power generation patterns vary, which may risk distribution network operations. So, it is intrinsically necessary to monitor wind turbines in a distributed way. This paper presents an adaptivethen-combine distributed dynamic approach for monitoring the grid under lossy communication links between the wind turbines and energy management system. Firstly, the wind turbine is represented by a state-space linear model, with sensors deployed to the obtain the system state information. Based on the mean squared error principle, an adaptive approach is proposed to estimate the local state information. The global estimation is designed by combining estimation results with weighting factors which are calculated by minimizing the estimation error covariance based on semidefinite programming. Finally, the convergence analysis indicates that the estimation error is gradually decreased, so the estimated state converges to the actual state. The efficacy of the developed approach is verified using the wind turbine and IEEE 6-bus distribution system.
\end{abstract}

Keywords-Adaptive-then-combine, Kalman filter, Lyapunov function, packet losses, semidefinite programming, wind turbine.

\section{INTRODUCTION}

Nowadays, the penetration of renewable distributed energy resources (DERs) such as wind turbine, tidal, and solar cells is radically increasing owing to the government incentives for low carbon emission strategies and meeting on-going energy demand [1], [2]. Among available renewable green resources, the wind turbine has relatively low investment cost and high generation capacity compared with solar panels and tidal systems [3]. So far, Denmark produces about 33\% electricity from wind farms and 50\% is targeted in 2020. The European Wind Energy Association set a target to achieve 25\% electricity from wind farms in 2030 [4]. However, the integration of wind turbines into the grid is associated with serious practical challenges, such as grid synchronization, power quality, stability, and reliability [5]. This is basically due to the fact that the wind speed generally depends on nature and meteorological conditions. Usually, the wind farm is situated near consumers or sea areas [6]. If the wind farm is installed in rural/sea areas, its operating condition should be closely monitored. State estimation for the microgrid incorporating wind turbines is a vital task in the grid energy management system (EMS).
Mostly, the wind firm and EMS is located far away from each other [6], so there is an unreliable communication link between them. [7] illustrates that the sensing measurements are affected by communication impairments when they are transmitted through an unreliable communication network. The unreliable link such as packet losses causes monitoring performance degradation and may give deceiving information to the utility operator [8], [9], [10]. A Kalman filter (KF) based state estimation via wireless sensor networks over fading channels and missing measurements are presented in [11], [12], [13]. Hence, the packet losses are considered as another form of power system contingency which may lead to a massive blackout if suitable actions from the operator are not taken on time. Therefore, state estimation considering packet losses has drawn significant attention and triggered a new wave of research on the smart distribution system [12], [13].

Research has been conducted on the power system state estimation in a centralized or distributed way. The centralized estimation means that the EMS uses all measurements from the local sensors to obtain a global estimation [14], [15], [16], thus demanding huge communication and computation resources for processing all the measurements. Firstly, all sensing measurements are transmitted to the central estimator, so more communication bandwidth is required compared with the distributed one. The distributed system can process the subset of sensing information in a distributed way, leading to a very effective strategy for performing the wide-area distributed computation [13], [17], [18]. Secondly, if there is problem in the central estimator and its may fail to process everything. This may lead to a single point failure or massive blackout. For instance, more than $95 \%$ of power outages [19] is owing to distribution system faults (excluding the reason of generation deficiency). In the distributed state estimation, every local estimator computes the state information based on its own measurements and sends it to the global estimator for obtaining a reliable estimation. From the smart grid and practical point of views, it improves scalability and computational efficiency [14], [15]. It is also relatively easier for monitoring the microgrid operating condition which changes after adding/removing the microgrid to/from the grid. There are several estimation methods that deals with the centralized and distributed state estimation. First of all, weighted least squares is a classic method for estimating power system states [20]. The distributed KF (DKF) based dynamic state estimation is 
widely used in the literature [21]. Moreover, the extended KF and unscented KF algorithms are proposed in [22]. Nowadays, the consensus-based DKF methods have been proposed for sensor networks in [23], [24]. From the practical point of view, the diffusion strategy is widely used in the literature, where the global estimator linearly combines local results using a set of weighing factors [25], [26]. However, selecting an optimal weighting factor from the power system perspective is a quite difficult task. Furthermore, none of the above papers consider the packet losses in the distributed estimation process. Interestingly, the KF and linear quadratic Gaussian based optimal control for the networked controlled smart grid is suggested in [12]. Even though it considers packet losses, it is only suitable for the centralized power system state estimation and control. In fact, the microgrid state estimation with an unreliable communication channel is not considered in the literature specifically in a distributed way.

This paper presents an adaptive-then-combine distributed dynamic approach for monitoring the wind turbine under packet loss conditions and it seems more preferred for many practical issues. After the local estimation, the global point fuses local results to yield an optimal system state estimation. The knowledge of state enables EMS to perform locally various crucial tasks, such as contingency analysis, bad data detection as well as maintaining system stability and reliability. The key contributions of the paper are as follows:

- Environment-friendly wind turbine is described as a linear state-space model, and the system measurements are obtained at EMS under unreliable communication links. The considered model and scenario are usually required in the power industry for the development of green technologies.

- In order to know the wind turbine operating conditions, an adaptive-then-combine distributed dynamic state estimation method is proposed. After locally estimating the system states, the global estimator combines local estimation results through a set of designed weighting factors. The weighting coefficients are calculated by minimizing estimation error covariances based on semidefinite programming (SDP).

- Convergence of the proposed approach is analyzed based on the Lyapunov approach. The proof indicates that the error function is a decreasing sequence, so that the error system is asymptotically stable. The analysis is significantly important for validation of the algorithm and simulation results.

The outline of this paper is as follows. A community resilient wind turbine state-space model is described in Section II, followed by the measurement model and problem formulation in Section III and IV, respectively. In Section V, an adaptive-then-combine diffusion algorithm and a weighting factor calculation method are proposed. Convergence of the proposed method is analyzed in Section VI. In Section VII, the developed approach is applied to the wind turbine and IEEE 6-bus distributed test system and the effectiveness of the proposed method is demonstrated. In Section VIII, the conclusion is drawn and recommendations are made for future directions of research.
Notation: Bold face lower and upper case letters are used to represent vectors and matrices, respectively. $E(\cdot)$ denotes the expectation operator, $\operatorname{tr}(\cdot)$ denotes the trace operator, $\mathbf{x}^{\prime}$ denotes the transpose of $\mathbf{x}$ and $\mathbf{I}$ denotes the identity matrix.

\section{WIND TURBINE MODEL}

Usually, wind turbines are the most important and promising DERs which are contributing considerably to the world's power generation [27]. The wind turbine converts the wind kinetic energy into electrical energy. In other words, the power generated by the wind turbine is transferred to the grid through inverter and step up transformer. To illustrate, the structure of a wind turbine is shown in Fig. 1 [28]. It consists of aerodynamic rotor, drive train (mechanical part) and generator (electrical part) [29]. The drive train is the connection between the rotor

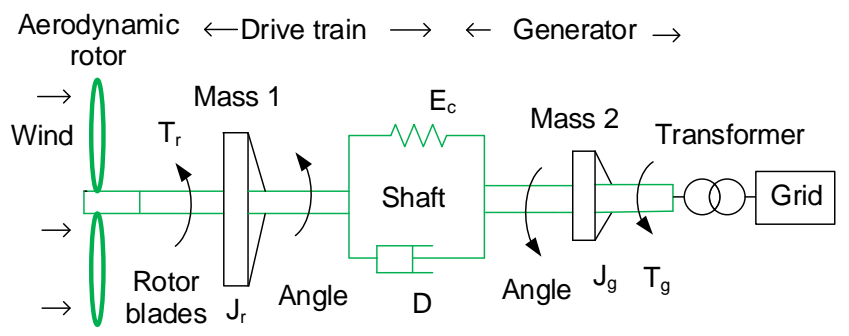

Fig. 1: Two-mass wind turbine model [28].

and generator. The rotor of the turbine is driven by blades. The blade converts the wind kinetic energy into torque. Then the drive train converts it into high rotational speed to drive the generator [28], [30]. Generally, wind turbines are designed to generate high torque at low speed due to the low wind speed and its huge variations. The turbine output power $P_{a}$ is determined as follows: [27]:

$$
P_{a}=\frac{1}{2} s \rho v^{3}=\frac{1}{2} \pi r^{2} \rho v^{3},
$$

where $s=\pi r^{2}$ is the area covered by rotor, $r$ is the rotor radius, $\rho$ is the air density, and $v$ is the wind speed. As the wind speed $v$ increases, the capturing power rises as roughly the cube of the speed. Normally, the power that can be converted by rotor is limited by the power coefficient [31], so the mechanical power $P_{e x t}$ extraction from the wind can be written as follows:

$$
P_{\text {ext }}=\frac{1}{2} s \rho c_{p}(\zeta, \beta) v^{3},
$$

where $\beta$ is the rotor blade pitch angle and $c_{p}(\zeta, \beta)$ is the power coefficient. The power coefficient is a nonlinear function of $\beta$ and tip speed ratio $\zeta$ i.e.,

$$
\zeta=\omega_{r} r / v
$$

where $\omega_{r}$ is the rotor angular rotational speed.

Mostly, the mechanical power generated by the wind turbine is transferred from the rotor to generator terminal. This connection is called drive train, and the dynamic model of drive 
train is represented by the following second order differential equations:

$$
\begin{aligned}
J_{r} \ddot{\theta}_{r} & =T_{r}-T_{s} . \\
J_{g} \ddot{\theta}_{g} & =T_{s}-T_{g} .
\end{aligned}
$$

Here, $J_{r}, T_{r}$, and $\theta_{r}$ are the moment of inertia, torque, and azimuth angle of the wind turbine. $J_{g}, T_{g}$, and $\theta_{g}$ are the moment of inertia, torque, and azimuth angle of the generator. As shown in Fig. 1 the shaft of the wind turbine is represented by a two-mass model, where the first mass represents a low speed turbine, and the second mass represents a high-speed generator. The two-mass connection is modelled as a spring and a damper [32], [33]. The equivalent shaft torque $T_{s}$ is given by:

$$
T_{s}=D\left(\dot{\theta}_{r}-\dot{\theta}_{g}\right)+E_{c}\left(\theta_{r}-\theta_{g}\right),
$$

where $D$ is the damping coefficient and $E_{c}$ is the spring elastic coefficient of the shaft. Perturbation in the shaft torque $\delta T_{s}$ can be expressed as follows:

$$
\delta T_{s}=D\left(\delta \dot{\theta}_{r}-\delta \dot{\theta}_{g}\right)+E_{c}\left(\delta \theta_{r}-\delta \theta_{g}\right) .
$$

Using the Tailor series, $T_{r}$ is expressed at the operating points $\omega_{r 0}, v_{0}$, and $\beta_{0}$ as follows:

$$
T_{r}\left(\omega_{r}, v, \beta\right)=T_{r}\left(\omega_{r 0}, v_{0}, \beta_{0}\right)+\delta T_{r},
$$

where $\delta T_{r}=\alpha \delta v+\zeta \delta w_{r}+\xi \delta \beta$ with $\alpha=\left.\frac{\partial T_{r}}{\partial v}\right|_{\left(\omega_{r 0}, v_{0}, \beta_{0}\right)}, \zeta=$ $\left.\frac{\partial T_{r}}{\partial \omega_{r}}\right|_{\left(\omega_{r 0}, v_{0}, \beta_{0}\right)}$, and $\xi=\left.\frac{\partial T_{r}}{\partial \beta}\right|_{\left(\omega_{r 0}, v_{0}, \beta_{0}\right)}$. The perturbation in rotor is expressed by the following second order differential equation:

$$
J_{r} \delta \ddot{\theta}_{r}=T_{r}+\delta T_{r}-T_{s}-\delta T_{s} .
$$

At the operating point, the perturbation in rotor and generator deviation are zero. Let $T_{r o}, T_{s 0}$, and $T_{g 0}$ are the rotor, shaft, and generator torque at the operating points, respectively. Overall, the system dynamic can be represented by the following differential equations [28]:

$$
\begin{aligned}
& J_{r} \delta \ddot{\theta}_{r}=\alpha \delta v+\zeta \delta \omega_{r}+\xi \delta \beta-D\left(\delta \dot{\theta}_{r}-\delta \dot{\theta}_{g}\right)-E_{c}\left(\delta \theta_{r}-\delta \theta_{g}\right) . \\
& J_{g} \delta \ddot{\theta}_{g}=D\left(\delta \dot{\theta}_{r}-\delta \dot{\theta}_{g}\right)+E_{c}\left(\delta \theta_{r}-\delta \theta_{g}\right)-\delta T_{g} .
\end{aligned}
$$

For the sake of simplicity, let's define $x_{1}=\delta \dot{\theta}_{r}=\delta \omega_{r}, x_{2}=$ $T_{d}=E_{c}\left(\delta \theta_{r}-\delta \theta_{g}\right)$, and $x_{3}=\delta \dot{\theta}_{g}=\delta \omega_{g}$, then the dynamic equations can be rewritten as follows:

$$
\begin{aligned}
& J_{r} \dot{x}_{1}=\alpha \delta v+\zeta x_{1}+\xi \delta \beta-D\left(x_{1}-x_{3}\right)-x_{2} . \\
& \dot{x}_{2}=E_{c}\left(x_{1}-x_{3}\right) . \\
& J_{g} \dot{x}_{3}=D\left(x_{1}-x_{3}\right)+x_{2}-\delta T_{g} .
\end{aligned}
$$

If the generator runs at the rated speed, $\delta T_{g}=0$, so the turbine model is expressed as a state-space linear equation as follows:

$$
\dot{\mathbf{x}}=\mathbf{A} \mathbf{x}+\mathbf{B} u+\mathbf{G} n \text {. }
$$

Here, the turbine system state $\mathbf{x}=\left[\begin{array}{lll}x_{1} & x_{2} & x_{3}\end{array}\right]^{\prime}=$ $\left[\delta \omega_{r}, T_{d}, \delta \omega_{g}\right]^{\prime}$, system input $u=\delta \beta, n=\delta v, \mathbf{A}=$
$\left[\begin{array}{ccc}(\zeta-D) / J_{r} & -1 / J_{r} & D / J_{r} \\ E_{c} & 0 & -E_{c} \\ D / J_{g} & 1 / J_{g} & -D / J_{g}\end{array}\right], \mathbf{B}=\left[\begin{array}{lll}\xi / J_{r} & 0 & 0\end{array}\right]^{\prime}$, and $\mathbf{G}=\left[\begin{array}{lll}\alpha / J_{r} & 0 & 0\end{array}\right]^{\prime}$. Now the wind turbine model is expressed as a discrete time state-space linear equation as follows:

$$
\mathbf{x}_{k+1}=\mathbf{A}_{d} \mathbf{x}_{k}+\mathbf{B}_{d} u_{k}+\mathbf{G}_{d} n_{k}
$$

where $\mathbf{A}_{d}=\mathbf{I}+\mathbf{A} \Delta t, \Delta t$ is the discretization step size parameter, $\mathbf{B}_{d}=\mathbf{B} \Delta t, \mathbf{G}_{d}=\mathbf{G} \Delta t$, and $\mathbf{n}_{k}$ is the zero mean process noise whose covariance matrix is $\mathbf{Q}_{k}$.

\section{Measurement system Model}

The proposed distributed estimation scheme considering packet losses is described in Fig. 2. In order to simplify the discussion, it is assumed there are $\mathrm{N}=4$ observation stations in the distribution power network ${ }^{1}$. The measurements of the

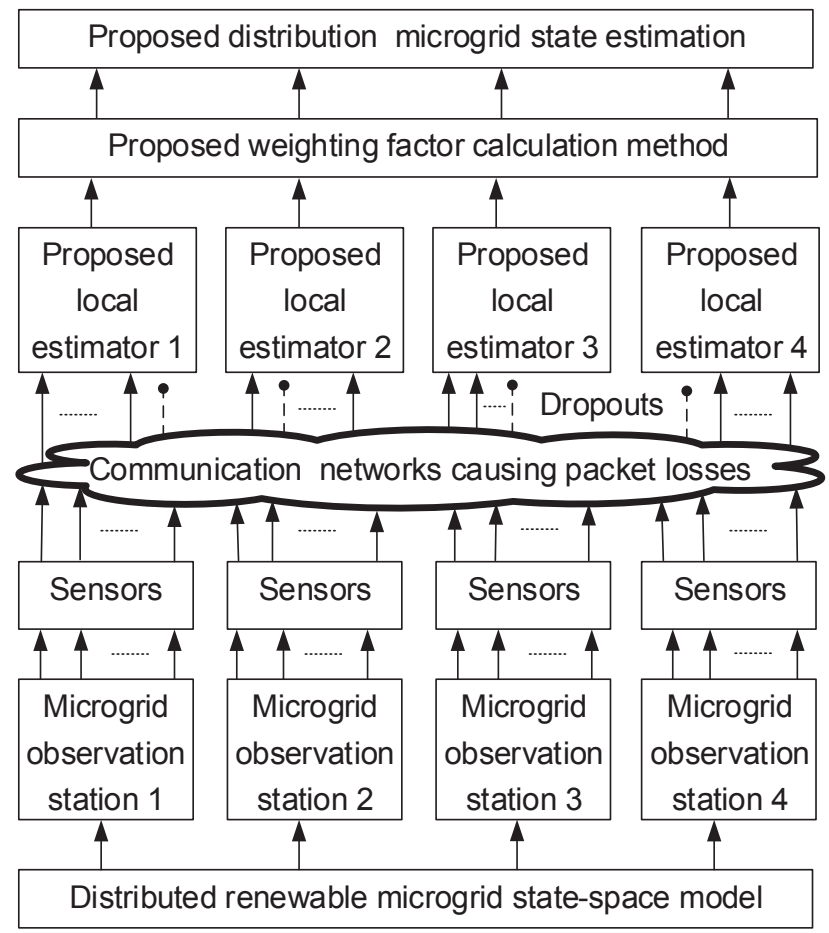

Fig. 2: Proposed distributed microgrid state estimation considering packet losses.

system are described by a set of sensors as follows:

$$
\mathbf{z}_{k}^{i}=\mathbf{C}^{i} \mathbf{x}_{k}+\mathbf{w}_{k}^{i},
$$

where $\mathbf{z}_{k}^{i}$ is the observation information for the i-th estimator at the time instant $\mathrm{k}, \mathbf{C}^{i}$ is the observation matrix, and $\mathbf{w}_{k}^{i}$ is the zero mean measurement noise whose covariance matrix is $\mathbf{R}_{k}^{i}$. Taking into account the packet loss, (17) can be written as follows:

$$
\mathbf{y}_{k}^{i}=\alpha_{k}^{i} \mathbf{C}^{i} \mathbf{x}_{k}+\alpha_{k}^{i} \mathbf{w}_{k}^{i},
$$

\footnotetext{
${ }^{1}$ The proposed work can be easily extended to the generic case.
} 
where $\mathbf{y}_{k}^{i}$ is the received measurements under the condition of packet losses, and $\alpha_{k}^{i} \in\{0,1\}$ is the Bernoulli distribution considered as a packet loss model in this work [12], [13]. Mathematically speaking, the packet loss can be modelled as follows:

$$
\alpha_{k}^{i}= \begin{cases}1, & \text { with probability of } \lambda_{k}^{i}, \\ 0, & \text { with probability of } 1-\lambda_{k}^{i},\end{cases}
$$

where $\lambda_{k}^{i}$ is the packet arrival rate reaching at the estimator.

Remark: The assumption of a Bernoulli packet loss model is inappropriate when the communication channel is congested [12], [34], [35]. In a congested channel the packet loss occurs in bursts, and follows a two-state Markov chain model, also known as Gilbert model [12], [34], [35].

\section{PROBLEM FORMULATION}

The local state estimation considering the packet losses is written as follows:

$$
\hat{\mathbf{x}}_{k \mid k}^{i}=\hat{\mathbf{x}}_{k \mid k-1}^{i}+\mathbf{K}_{k}^{i}\left[\mathbf{y}_{k}^{i}-\alpha_{k}^{i} \mathbf{C}^{i} \hat{\mathbf{x}}_{k \mid k-1}^{i}\right] .
$$

Here, $\hat{\mathbf{x}}_{k \mid k}^{i}$ is the local updated state estimation, $\hat{\mathbf{x}}_{k \mid k-1}^{i}$ is the predicted state estimation and $\mathbf{K}_{k}^{i}$ is the gain. The predicted state and error covariance are computed as follows:

$$
\begin{aligned}
& \hat{\mathbf{x}}_{k \mid k-1}^{i}=\mathbf{A}_{d} \hat{\mathbf{x}}_{k-1 \mid k-1}^{i}+\mathbf{B}_{d} u_{k} . \\
& \mathbf{P}_{k \mid k-1}^{i}=\mathbf{A}_{d} \mathbf{P}_{k-1 \mid k-1}^{i} \mathbf{A}_{d}^{\prime}+\mathbf{G}_{d} \mathbf{Q}_{k}^{i} \mathbf{G}_{d}^{\prime} .
\end{aligned}
$$

Here, $\hat{\mathbf{x}}_{k-1 \mid k-1}^{i}$ is the estimated state of the previous step and $\mathbf{P}_{k-1 \mid k-1}^{i}$ is the error covariance matrix of the previous step. Based on the aforementioned filtering process, our first problem is to design the optimal gain $\mathbf{K}_{k}^{i}$, so that the estimated state converges to the actual state. The second objective is to find a set of optimal weighting factors so that an accurate state estimation can be obtained by optimally combining the weighted local estimations. Our final objective is to analysis the convergence of the proposed state estimation method, so that the developed approach can apply to the real-time applications. Driven by the aforementioned motivations, the proposed scheme is demonstrated in Fig. 3. This paper tries

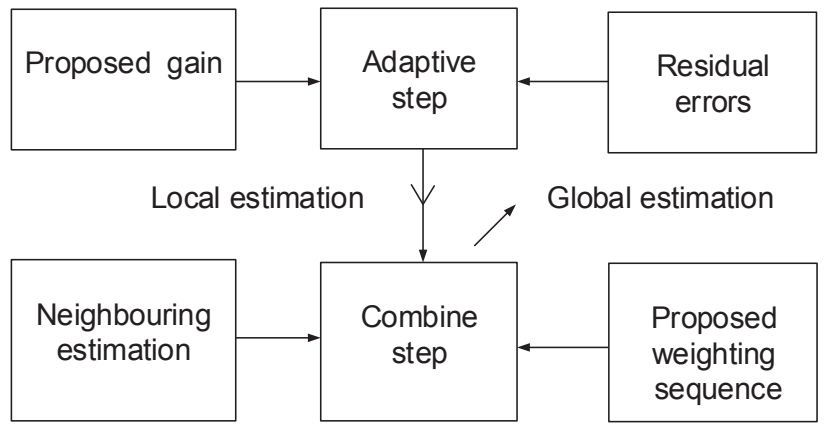

Fig. 3: Problem formulation and proposed strategy.

to answer these questions by presenting an adaptive-thencombine distributed dynamic algorithm in the subsequent sections.

\section{PROPOSED AdAPTIVE-THEN-COMBINE DISTRIBUTED DYNAMIC APPROACH}

Let $\mathbf{e}^{i}$ denotes the estimation error between the actual system state and estimated state of the i-th estimator, which can be expressed as follows:

$$
\begin{aligned}
& \mathbf{e}_{k \mid k-1}^{i}=\mathbf{x}_{k}-\hat{\mathbf{x}}_{k \mid k-1}^{i} . \\
& \mathbf{e}_{k \mid k}^{i}=\mathbf{x}_{k}-\hat{\mathbf{x}}_{k \mid k}^{i} .
\end{aligned}
$$

Now substituting (19) into (23), one can obtain the following expression:

$$
\begin{aligned}
\mathbf{e}_{k \mid k}^{i} & =\mathbf{x}_{k}-\hat{\mathbf{x}}_{k \mid k-1}^{i}-\mathbf{K}_{k}^{i}\left[\mathbf{y}_{k}^{i}-\alpha_{k}^{i} \mathbf{C}^{i} \hat{\mathbf{x}}_{k \mid k-1}^{i}\right] \\
& =\mathbf{x}_{k}-\hat{\mathbf{x}}_{k \mid k-1}^{i}-\mathbf{K}_{k}^{i}\left[\alpha_{k}^{i} \mathbf{C}^{i} \mathbf{x}_{k}+\alpha_{k}^{i} \mathbf{w}_{k}^{i}-\alpha_{k}^{i} \mathbf{C}^{i} \hat{\mathbf{x}}_{k \mid k-1}^{i}\right] \\
& =\left[\mathbf{I}-\alpha_{k}^{i} \mathbf{K}_{k}^{i} \mathbf{C}^{i}\right]\left[\mathbf{x}_{k}-\hat{\mathbf{x}}_{k \mid k-1}^{i}\right]-\alpha_{k}^{i} \mathbf{K}_{k}^{i} \mathbf{w}_{k}^{i} \\
& =\left[\mathbf{I}-\alpha_{k}^{i} \mathbf{K}_{k}^{i} \mathbf{C}^{i}\right] \mathbf{e}_{k \mid k-1}^{i}-\alpha_{k}^{i} \mathbf{K}_{k}^{i} \mathbf{w}_{k}^{i} .
\end{aligned}
$$

Now, the estimation error covariance matrix is defined by:

$$
\mathbf{P}_{k \mid k}^{i}=E\left[\mathbf{e}_{k \mid k}^{i} \mathbf{e}_{k \mid k}^{i}\right] .
$$

By substituting (24) into (25), one can obtain the following estimation error covariance matrix as follows:

$$
\begin{aligned}
\mathbf{P}_{k \mid k}^{i}= & \lambda_{k}^{i}\left[\mathbf{I}-\mathbf{K}_{k}^{i} \mathbf{C}^{i}\right] \mathbf{P}_{k \mid k-1}^{i}\left[\mathbf{I}-\mathbf{K}_{k}^{i} \mathbf{C}^{i}\right]^{\prime}+\lambda_{k}^{i} \mathbf{K}_{k}^{i} \mathbf{R}_{k}^{i} \mathbf{K}_{k}^{i}+ \\
& \left(1-\lambda_{k}^{i}\right) \mathbf{P}_{k \mid k-1}^{i} .
\end{aligned}
$$

For any two compatible matrices $\mathbf{X}$ and $\mathbf{Y}$, the following partial derivatives are holds:

$$
\begin{aligned}
& \frac{\partial \operatorname{tr}(\mathbf{Y X})}{\partial \mathbf{X}}=\mathbf{Y}^{\prime} . \\
& \frac{\partial \operatorname{tr}\left(\mathbf{X Y \mathbf { X } ^ { \prime }}\right)}{\partial \mathbf{X}}=\mathbf{X}\left(\mathbf{Y}+\mathbf{Y}^{\prime}\right)
\end{aligned}
$$

Taking the partial derivative of (26) with respect to $\mathbf{K}_{k}^{i}$ and applying (27) and (28) yields:

$$
\begin{aligned}
\frac{\partial\left[\operatorname{tr} \mathbf{P}_{k \mid k}^{i}\right]}{\partial \mathbf{K}_{k}^{i}}=- & 2 \lambda_{k}^{i} \mathbf{P}_{k \mid k-1}^{i} \mathbf{C}^{\prime i}+2 \lambda_{k}^{i} \mathbf{K}_{k}^{i} \mathbf{C}^{i} \mathbf{P}_{k \mid k-1}^{i} \mathbf{C}^{\prime i}+ \\
& 2 \lambda_{k}^{i} \mathbf{K}_{k}^{i} \mathbf{R}_{k}^{i} .
\end{aligned}
$$

Setting $\frac{\partial\left[t r \mathbf{P}_{k \mid k}^{i}\right]}{\partial \mathbf{K}_{k}^{i}}=\mathbf{0}$ and the optimal gain is given by:

$$
\mathbf{K}_{k}^{i}=\mathbf{P}_{k \mid k-1}^{i} \mathbf{C}^{\prime}{ }^{i}\left[\mathbf{C}^{i} \mathbf{P}_{k \mid k-1}^{i} \mathbf{C}^{\prime i}+\mathbf{R}_{k}^{i}\right]^{-1} .
$$

Next, we design an optimal linear estimator to fuse the local state estimations as follows:

$$
\hat{\mathbf{x}}_{k \mid k}^{g}=\sum_{i=1}^{N} w_{k}^{(i)} \hat{\mathbf{x}}_{k \mid k}^{i}
$$

Here, $\hat{\mathbf{x}}_{k \mid k}^{i}$ is the local estimation and $w_{k}^{(i)}$ is the weighting factor to be designed. It can be observed that designing an optimal set of weighing factors plays a vital role for the distributed state estimation in the context of smart grids. In 
order to find the optimal weighting factors, we propose the following (assuming $N=4$ ):

minimise $s$

$$
\begin{aligned}
& \text { subject to }(\left.w_{k}^{(1)}\right)^{2} d_{k \mid k}^{(1)}+\left(w_{k}^{(2)}\right)^{2} d_{k \mid k}^{(2)}+ \\
&\left(w_{k}^{(3)}\right)^{2} d_{k \mid k}^{(3)}+\left(w_{k}^{(4)}\right)^{2} d_{k \mid k}^{(4)} \leq s, \\
& w_{k}^{(1)}+w_{k}^{(2)}+w_{k}^{(3)}+w_{k}^{(4)}=1,
\end{aligned}
$$

where $d_{k \mid k}^{(i)}=\operatorname{tr}\left(\mathbf{P}_{k \mid k}^{i}\right)$, and $s$ is an auxiliary variable for minimizing the trace of the global estimator error covariance. Consequently, the error covariance is minimized, so that the estimated states match the true states. By using the semidefinite programming (SDP) variable $s$, the distributed estimation problem is converted to a convex problem which can be solved effectively and efficiently. Based on Schur's complement, (32) can be formulated as a linear matrix inequality:

$$
\left[\begin{array}{ccccc}
-s & w_{k}^{(1)} & w_{k}^{(2)} & w_{k}^{(3)} & w_{k}^{(4)} \\
w_{k}^{(1)} & -\left(d_{k \mid k}^{(1)}\right)^{-1} & 0 & 0 & 0 \\
w_{k}^{(2)} & 0 & -\left(d_{k \mid k}^{(2)}\right)^{-1} & 0 & 0 \\
w_{k}^{(3)} & 0 & 0 & -\left(d_{k \mid k}^{(3)}\right)^{-1} & 0 \\
w_{k}^{(4)} & 0 & 0 & 0 & -\left(d_{k \mid k}^{(4)}\right)^{-1}
\end{array}\right] \leq 0 .
$$

Finally, one can formulate the proposed optimization problem as follows:

$$
\begin{array}{ll}
\text { minimise } & s \\
\text { subject to } H o l d & (33), \text { and }(34) .
\end{array}
$$

In summary, the proposed distributed state estimation algorithm in the context of smart grids is summarized in Table I. The main advantage of this method is that it only requires

TABLE I: Proposed distributed state estimation algorithm.

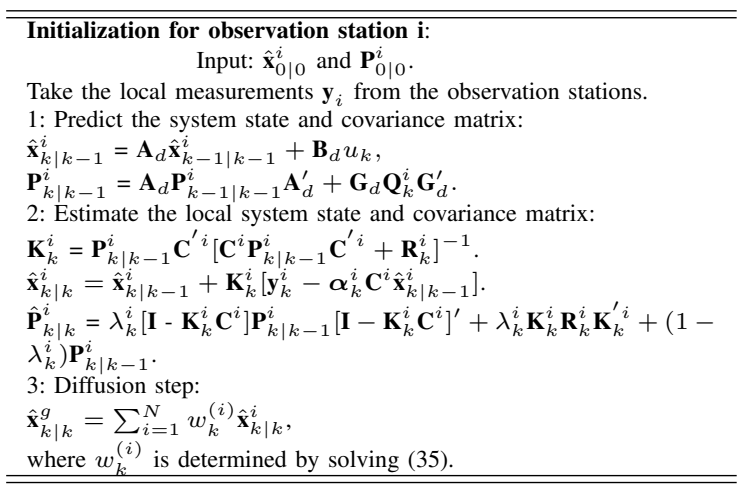

low computation complexity and communication resources. It also does not need neighborhood communication in contrast to consensus algorithm [36] while maintaining accurate and consistent estimation. Even though there are missing observations, the optimization method can improve the accurate and consistent estimation after selecting suitable weighting coefficients.
This is due to the fact that it runs in an automated way and is well adapted to the unknown change in measurements. Now the question is that how we can guarantee the convergence of the proposed state estimation method.

\section{CONVERGENCE ANALYSIS}

From the engineering perspective, the discrete-time system is easy to implement in the digital platforms, while the continuous system is easy to analyze from the mathematical point of view [37]. Motivated by this realistic dilemma and similar to [23], the convergence analysis of the adaptive-thencombine algorithm is completed based on the convergence analysis of the continuous system. Similar to the discrete-time system, the estimator applies the following step:

$$
\dot{\hat{\mathbf{x}}}^{i}=\mathbf{A} \hat{\mathbf{x}}^{i}+\mathbf{B} u+\mathbf{K}^{i}\left[\mathbf{y}^{i}-\boldsymbol{\alpha}^{i} \mathbf{C}^{i} \hat{\mathbf{x}}^{i}\right] .
$$

The estimation error $\mathbf{e}^{i}$ can be expressed as follows:

$$
\mathbf{e}^{i}=\mathbf{x}-\hat{\mathbf{x}}^{i}
$$

By direct differentiating (37), with (15) and (18), the estimation error dynamics is in the following form:

$$
\begin{aligned}
& \dot{\mathbf{e}^{i}}=\dot{\mathbf{x}}-\dot{\hat{\mathbf{x}}}^{i} \\
& =\mathbf{A x}+\mathbf{B} u+\mathbf{G} n-\mathbf{A} \hat{\mathbf{x}}^{i}-\mathbf{B} u-\alpha^{i} \mathbf{K}^{i}\left[\mathbf{C}^{i} \mathbf{x}+\mathbf{w}^{i}-\mathbf{C}^{i} \hat{\mathbf{x}}^{i}\right] \\
& =\left(\mathbf{A}-\alpha^{i} \mathbf{K}^{i} \mathbf{C}^{i}\right) \mathbf{e}^{i}+\mathbf{G} n-\alpha^{i} \mathbf{K}^{i} \mathbf{w}^{i} .
\end{aligned}
$$

The error covariance matrix under the condition of losses is written as follows:

$$
\begin{aligned}
\dot{\mathbf{P}^{i}} & =\left(\mathbf{A}-\lambda^{i} \mathbf{K}^{i} \mathbf{C}^{i}\right) \mathbf{P}^{i}+\mathbf{P}^{i}\left(\mathbf{A}-\lambda^{i} \mathbf{K}^{i} \mathbf{C}^{i}\right)^{\prime}+\mathbf{G} \mathbf{Q} \mathbf{G}^{\prime}+\lambda^{i} \mathbf{K}^{i} \mathbf{R}^{i} \mathbf{K}^{i} \\
& =\mathbf{A} \mathbf{P}^{i}+\mathbf{P}^{i} \mathbf{A}^{\prime}+\mathbf{G} \mathbf{Q} \mathbf{G}^{\prime}-\lambda^{i} \mathbf{K}^{i} \mathbf{C}^{i} \mathbf{P}^{i}-\lambda^{i} \mathbf{P}^{i} \mathbf{C}^{i} \mathbf{K}^{\prime i}+\lambda^{i} \mathbf{K}^{i} \mathbf{R}^{i} \mathbf{K}^{\prime i} .
\end{aligned}
$$

Taking the partial derivative of (39) with respect to $\mathbf{K}^{i}$ and applying (27) and (28) yields:

$$
\frac{\partial\left[\operatorname{tr} \dot{\mathbf{P}}^{i}\right]}{\partial \mathbf{K}^{i}}=-2 \lambda^{i} \mathbf{P}^{i} \mathbf{C}^{i}+2 \lambda^{i} \mathbf{K}^{i} \mathbf{R}^{i} .
$$

Setting $\frac{\partial\left[\operatorname{tr} \dot{\mathbf{P}}^{i}\right]}{\partial \mathbf{K}^{i}}=\mathbf{0}$ and the gain matrix is given by:

$$
\mathbf{K}^{i}=\mathbf{P}^{i} \mathbf{C}^{i}\left(\mathbf{R}^{i}\right)^{-1} .
$$

Substituting (41) into (39), one can obtain the following error covariance matrix:

$$
\begin{aligned}
\dot{\mathbf{P}^{i}}= & \mathbf{A} \mathbf{P}^{i}+\mathbf{P}^{i} \mathbf{A}^{\prime}+\mathbf{G Q G} \mathbf{G}^{\prime}-\lambda^{i} \mathbf{P}^{i} \mathbf{C}^{i}\left(\mathbf{R}^{i}\right)^{-1} \mathbf{C}^{i} \mathbf{P}^{i}- \\
& \lambda^{i} \mathbf{P}^{i} \mathbf{C}^{\prime i}\left(\mathbf{R}^{i}\right)^{-1} \mathbf{C}^{i} \mathbf{P}^{i}+\lambda^{i} \mathbf{P}^{i} \mathbf{C}^{\prime i}\left(\mathbf{R}^{i}\right)^{-1} \mathbf{C}^{i} \mathbf{P}^{i} \\
= & \mathbf{A} \mathbf{P}^{i}+\mathbf{P}^{i} \mathbf{A}^{\prime}+\mathbf{G Q G} \mathbf{G}^{\prime}-\lambda^{i} \mathbf{P}^{i} \mathbf{C}^{\prime i}\left(\mathbf{R}^{i}\right)^{-1} \mathbf{C}^{i} \mathbf{P}^{i}
\end{aligned}
$$

In order to analyze the stability of the proposed approach, defining $\overline{\mathbf{e}}^{i}=E\left(\mathbf{e}^{i}\right)$, and taking the expectation on both sides of (38), we have:

$$
\dot{\overline{\mathbf{e}}}^{i}=\left(\mathbf{A}-\lambda^{i} \mathbf{K}^{i} \mathbf{C}^{i}\right) \overline{\mathbf{e}}^{i} .
$$


Consider the following Lyapunov function:

$$
V=\sum_{i=1}^{N} \overline{\mathbf{e}}^{i}\left(\mathbf{P}^{i}\right)^{-1} \overline{\mathbf{e}}^{i} .
$$

Now taking the partial derivative and expectation of (44). Using (41), (42) and (43), the Lyapunov function can be written as follows:

$$
\begin{aligned}
\dot{V}= & \sum_{i=1}^{N}\left\{\dot{\mathbf{e}}^{\prime}\left(\mathbf{P}^{i}\right)^{-1} \overline{\mathbf{e}}^{i}+\overline{\mathbf{e}}^{i}\left(\mathbf{P}^{i}\right)^{-1} \dot{\mathbf{e}}^{i}-\overline{\mathbf{e}}^{i}\left(\mathbf{P}^{i}\right)^{-1} \dot{\mathbf{P}}^{i}\left(\mathbf{P}^{i}\right)^{-1} \overline{\mathbf{e}}^{i}\right\} \\
= & \sum_{i=1}^{N} \overline{\mathbf{e}}^{\prime}\left[-\lambda^{i} \mathbf{C}^{i}\left(\mathbf{R}^{i}\right)^{-1} \mathbf{C}^{i}-\lambda^{i} \mathbf{C}^{\prime i}\left(\mathbf{R}^{i}\right)^{-1} \mathbf{C}^{i}-\right. \\
& \left.\left(\mathbf{P}^{i}\right)^{-1} \mathbf{G Q G} \mathbf{G}^{\prime}\left(\mathbf{P}^{i}\right)^{-1}+\lambda^{i} \mathbf{C}^{\prime i}\left(\mathbf{R}^{i}\right)^{-1} \mathbf{C}^{i}\right] \overline{\mathbf{e}}^{i} \\
= & \left.-\sum_{i=1}^{N} \overline{\mathbf{e}}^{\prime} i \lambda^{i} \mathbf{C}^{i}\left(\mathbf{R}^{i}\right)^{-1} \mathbf{C}^{i}+\left(\mathbf{P}^{i}\right)^{-1} \mathbf{G} \mathbf{Q G} \mathbf{G}^{\prime}\left(\mathbf{P}^{i}\right)^{-1}\right] \overline{\mathbf{e}}^{i}<0 .
\end{aligned}
$$

The inequality (45) shows that the Lyapunov function is gradually decreased, so the error function (43) is asymptotically stable. Consequently, the estimated state $\hat{\mathbf{x}}^{i}$ converges to the actual system state $\mathbf{x}$. This concludes the proposed filtering algorithm is convergent, which is significantly important for real-time applications.

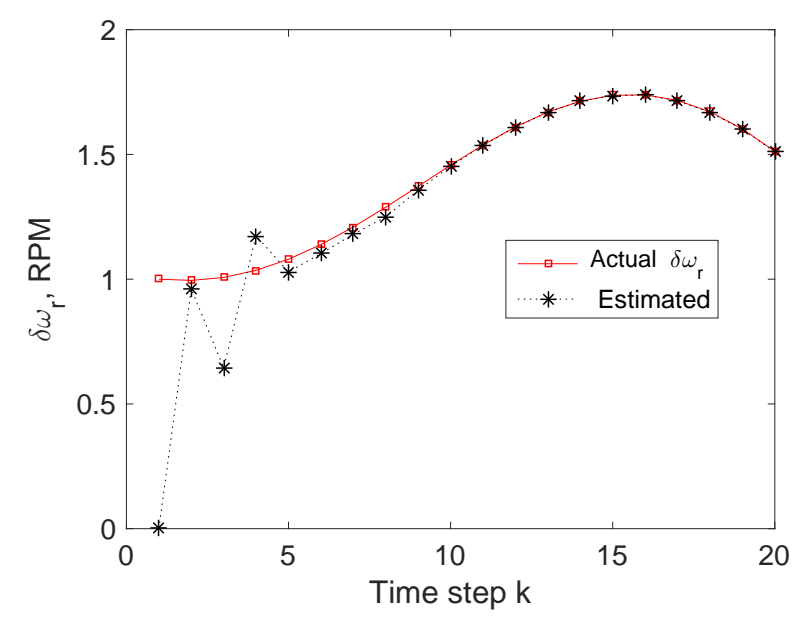

Fig. 4: Rotor speed deviation $\delta \omega_{r}$ and its estimation.

\section{CASE STUDIES AND DISCUSSION}

In this section, the proposed algorithm is tested and the results are demonstrated using the wind turbine (located in the distribution system) and IEEE 6-bus distribution system, respectively. It is assumed that the complete system state could not be measured directly, and the measurement of each sensor is different. The system parameters are shown in Table II [28], [30]. The simulation has been carried out using the Matlab, Matpower [38], and YALMIP softwares [39].

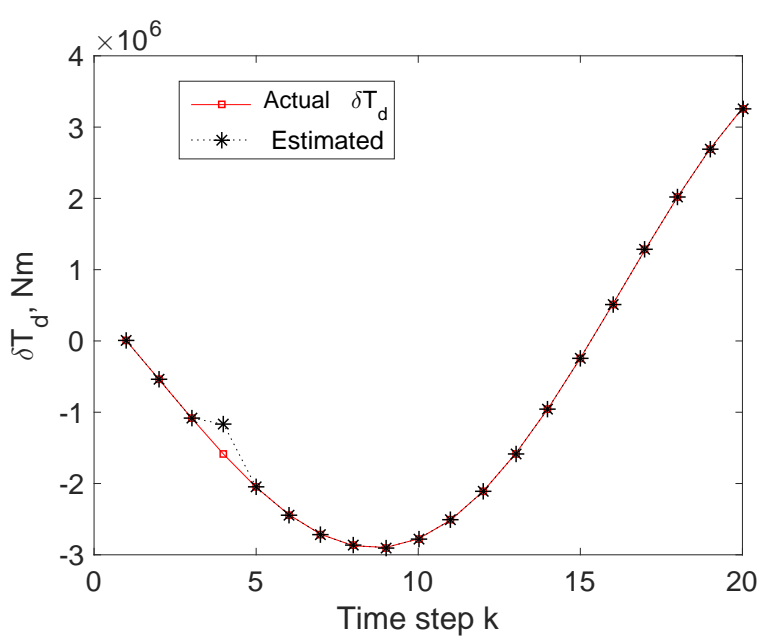

Fig. 5: Drive-train torsional spring force deviation $\delta T_{d}$ and its estimation.

TABLE II: Simulation parameters.

\begin{tabular}{cccc}
\hline Symbols & Values & Symbols & Values \\
\hline$\omega_{\text {ro }}$ & $42 \mathrm{RPM}$ & $v_{0}$ & $18 \mathrm{~m} / \mathrm{s}$ \\
$\beta_{0}$ & $12 \mathrm{deg}$ & $E_{c}$ & $2.691 \times 10^{7} \mathrm{Nm} /$ deg. \\
$\mathrm{r}$ & $21.65 \mathrm{~m}$ & $\mathrm{D}$ & $0 \mathrm{Nm} / \mathrm{deg}-\mathrm{s}$ \\
$J_{r}$ & $3.2175 \times 10^{5}$ & $J_{g}$ & $6.4103 \times 10^{4}$ \\
$\Delta t$ & 0.01 & $\lambda^{1}$ & 0.93 \\
$\lambda^{2}$ & 0.95 & $\lambda^{3}$ & 0.98 \\
$\lambda^{4}$ & 0.97 & $\mathbf{R}^{1}$ & $0.0001^{*} \mathbf{I}$ \\
$\mathbf{R}^{2}$ & $0.00012^{*} \mathbf{I}$ & $\mathbf{R}^{3}$ & $0.00014^{*} \mathbf{I}$ \\
$\mathbf{R}^{4}$ & $0.00015^{*} \mathbf{I}$ & $\mathbf{Q}^{i}$ & $0.00001 * \mathbf{I}$ \\
\hline
\end{tabular}

\section{A. Results based on the wind turbine}

Figures 4-6 show the system states versus time step for the wind turbine. It can be seen that the proposed method can estimate the turbine states with reasonable accuracy. This is because the local estimators are able to track the system states after rejecting the statistical impairments as much as possible. In other words, the mean square error based designed gain provides accurate influence to remove the system impairments. Then the global estimator properly calculates the optimal weighting factors by minimizing the estimated error covariances. Consequently, the fusion estimator can greatly reduce the global estimation error, so the estimated state converges to the true system state. Basically, the proposed SDP based estimator can efficiently solve the distributed state estimation problem to find the optimal solution. It can be seen from Fig. 4 that the proposed method requires maximum 0.1 second $(k \times \Delta t=10 \times 0.01)$ to estimate the system state. Technically, it means that the developed approach requires much less time compared with the standard estimation time of 1 second [40]. Note that the small fluctuations come from system impairments, but it does not affect the estimation accuracy. In other words, the estimation result would not mislead the utility operator even though there is missing measurements. As this is an open-loop system without control, the system dynamics 


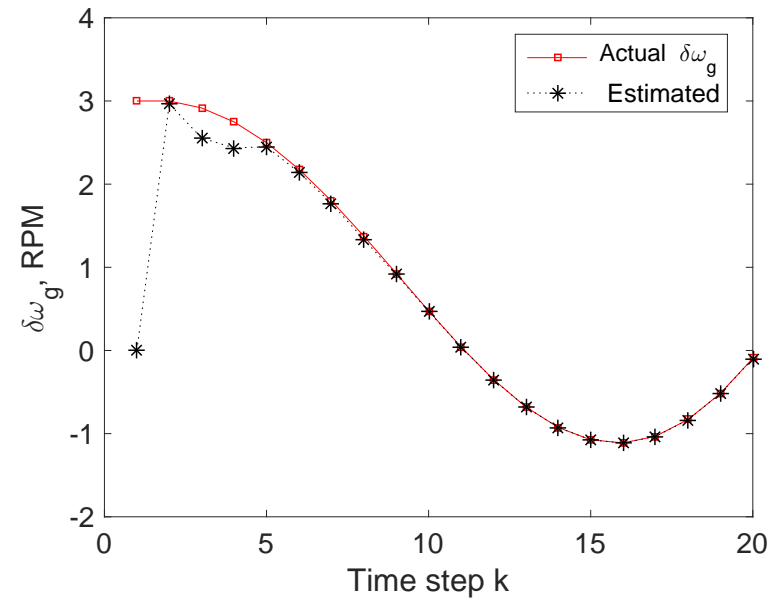

Fig. 6: Generator speed deviation $\delta \omega_{g}$ and its estimation.

cannot be guaranteed to be stable. As can be seen in the simulation results, the state will fluctuate which is determined by the open-loop system $\mathbf{A}$ matrix.

\section{B. Estimation results using the IEEE 6-bus test system}

The IEEE 6-bus testing system is employed to demonstrate the performance of the proposed approach. A single-line diagram of the IEEE 6-bus distribution test system is depicted in Fig. 7. The system has three generators on buses 1, 2 and 6

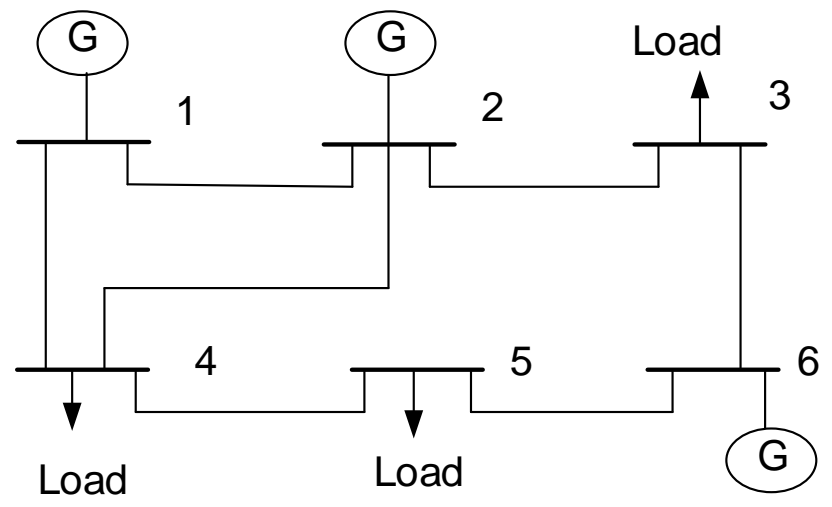

Fig. 7: Single-line diagram of the IEEE 6-bus power distribution system [41].

and three loads on buses 3, 4 and 5. It has the total generation capacity $217.88 \mathrm{MW}$ and load capacity $210 \mathrm{MW}$. The nominal phase angles and bus voltage magnitudes are shown in Table III.

Similarly, it is evident from the dynamic responses in Figs. 8-9 that the estimation results match the actual system states within few steps. This clearly implies that the explored method can well reject the system impairments, and accurately monitor the system states. To sum up, this paper has presented an
TABLE III: The nominal values of the IEEE 6-bus system.

\begin{tabular}{cccccc} 
Bus & $\Theta_{N}$ & $V_{N}$ & Bus & $\Theta_{N}$ & $V_{N}$ \\
\hline 1 & 0 & 1.050 & 4 & -4.196 & 0.989 \\
2 & -3.671 & 1.050 & 5 & -5.276 & 0.985 \\
3 & -4.273 & 1.070 & 6 & -5.947 & 1.004 \\
\hline
\end{tabular}

adaptive-then-combine distributed dynamic state estimation algorithm, fundamental results, convergence analysis, and application to the emerging smart grid communication area.

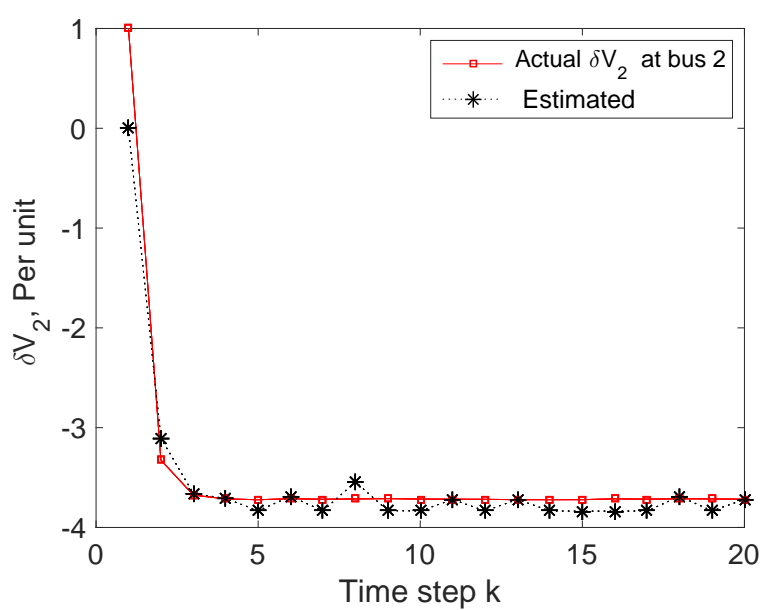

Fig. 8: Voltage deviation $\delta V_{2}$ and its estimation using the IEEE 6-bus.

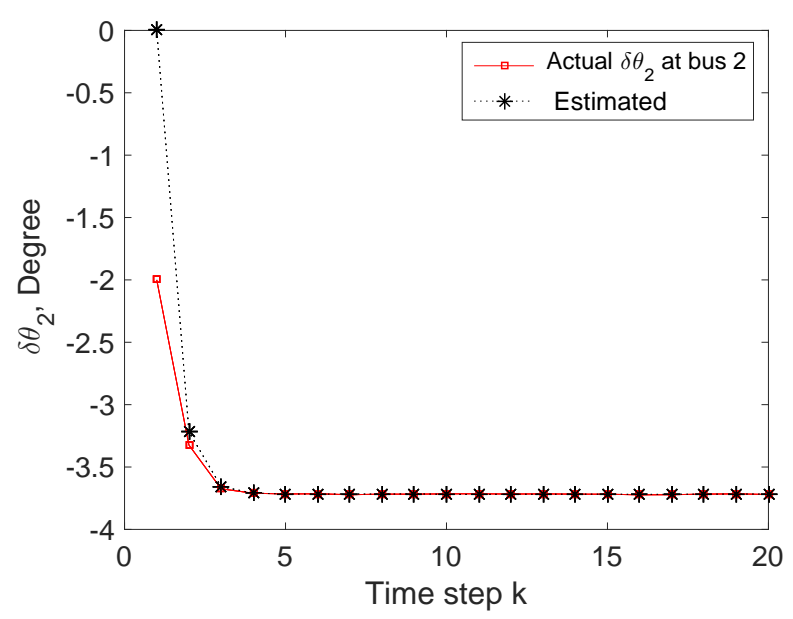

Fig. 9: Phase angle deviation $\delta \theta_{2}$ and its estimation using the IEEE 6-bus.

\section{CONCLUSION AND FUTURE WORK}

The increased penetration of renewable energy presents a series of technical challenges in power system operations. To 
monitor such a system under lossy network conditions, we propose an adaptive-then-combine distributed dynamic state estimation algorithm based on the mean squared error and SDP approaches. After estimating the local information, the global estimator combines the locally estimated results with a set of weighting factors, which are calculated by the proposed convex optimization algorithm. The theoretical convergence analysis is also verified through numerical simulation results. So, these findings are valuable for green communication, households, and provides the knowledge towards smart EMS design. Although our proposed system model and algorithm are useful for smart grid applications, the work presented here has some limitations. Therefore, further investigations will include the following aspects:

- Test the proposed estimation algorithm considering more dynamic conditions such as a step, an inrush and a sudden power injection.

- The suggested approach requires statistical information about the process and measurement noises which are often unavailable in practice. In light of the problem, distributed $H_{\infty}$ filtering is the potential method.

- Future work also includes applying the distributed state estimation algorithm to the large-scale power systems with disturbances, delay, and cyber attacks.

\section{REFERENCES}

[1] M. Sedghi, A. Ahmadian, and M. Aliakbar-Golkar, "Optimal storage planning in active distribution network considering uncertainty of wind power distributed generation," IEEE Transactions on Power Systems, vol. 31, no. 1, pp. 304-316, 2016.

[2] P. Xiong and D. Sun, "Backstepping-based DPC strategy of wind turbine driven DFIG under normal and harmonic grid voltage," IEEE Transactions on Power Electronics, vol. 31, no. 6, pp. 4216-4225, 2016.

[3] C.-L. Nguyen, H.-H. Lee, and T.-W. Chun, "Cost-optimized battery capacity and short-term power dispatch control for wind farm," IEEE Transactions on Industry Applications, vol. 51, no. 1, pp. 595-606, 2015.

[4] Z. H. Rather, Z. Chen, P. Thogersen, and P. Lund, "Dynamic reactive power compensation of large-scale wind integrated power system," IEEE Transactions on Power Systems, vol. 30, no. 5, pp. 2516-2526, 2015.

[5] L. P. Kunjumuhammed, B. C. Pal, C. Oates, and K. J. Dyke, "Electrical oscillations in wind farm systems: Analysis and insight based on detailed modeling," IEEE Transactions on Sustainable Energy, vol. 7, no. 1, pp. 51-62, 2016.

[6] X. Li and H. Gao, "Load mitigation for a floating wind turbine via generalized structural control," IEEE Transactions on Industrial Electronics, vol. 63, no. 1, pp. 332-342, 2016.

[7] L. Schenato, "Optimal estimation in networked control systems subject to random delay and packet drop," IEEE Transactions on Automatic Control, vol. 53, no. 5, pp. 1311-1317, 2008.

[8] S. Deshmukh, B. Natarajan, and A. Pahwa, "State estimation over a lossy network in spatially distributed cyber-physical systems," IEEE Transactions on Signal Processing, vol. 62, no. 15, pp. 3911-3923, 2014.

[9] B. Sinopoli, L. Schenato, M. Franceschetti, K. Poolla, M. Jordan, and S. Sastry, "Kalman filtering with intermittent observations," IEEE Transactions on Automatic Control, vol. 49, no. 9, pp. 1453-1464, 2004.

[10] A. Abdrabou and A. M. Gaouda, "Uninterrupted wireless data transfer for smart grids in the presence of high power transients," IEEE System Journal.
[11] D. E. Quevedo and A. Ahlen, "A predictive power control scheme for energy efficient state estimation via wireless sensor networks," in Proc. of the Decision and Control, 2008, pp. 1103-1108.

[12] A. K. Singh, R. Singh, and B. C. Pal, "Stability analysis of networked control in smart grids," IEEE Transactions on Smart Grid, vol. 6, no. 1, pp. 381-390, 2015.

[13] L. Hu, Z. Wang, I. Rahman, and X. Liu, "A constrained optimization approach to dynamic state estimation for power systems including PMU and missing measurements," IEEE Transactions on Control Systems Technology, vol. 24, no. 2, pp. 703-710, 2016.

[14] E. Song, J. Xu, and Y. Zhu, "Optimal distributed Kalman filtering fusion with singular covariances of filtering errors and measurement noises," IEEE Transactions on Automatic Control, vol. 59, no. 5, pp. 1271-1282, 2014.

[15] M. J. Daigle, A. Bregon, and I. Roychoudhury, "Distributed prognostics based on structural model decomposition," IEEE Transactions on Reliability, vol. 63, no. 2, pp. 495-510, 2014.

[16] D. E. Olivares, C. A. Cañizares, and M. Kazerani, "A centralized energy management system for isolated microgrids," IEEE Transactions on Smart Grid, vol. 5, no. 4, pp. 1864-1875, 2014.

[17] M. Tahir and S. K. Mazumder, "Self-triggered communication enabled control of distributed generation in microgrids," IEEE Transactions on Industrial Informatics, vol. 11, no. 2, pp. 441-449, 2015.

[18] H. Li and Z. Han, "Distributed scheduling of wireless communications for voltage control in micro smart grid," in Proc. of the GLOBECOM Workshops, 2011, pp. 1188-1193.

[19] H. Sui and W.-J. Lee, "An AMI based measurement and control system in smart distribution grid," in Proc of the Industrial and Commercial Power Systems Technical Conference, 2011, pp. 1-5.

[20] L. Xie, D. H. Choi, S. Kar, and H. V. Poor, "Fully distributed state estimation for wide-area monitoring systems," IEEE Transactions on Smart Grid, vol. 3, no. 3, pp. 1154-1169, 2012.

[21] H. R. Hashemipour, S. Roy, and A. J. Laub, "Decentralized structures for parallel Kalman filtering," IEEE Transactions on Automatic Control, vol. 33, no. 1, pp. 88-94, 1988.

[22] S.-C. Huang, C.-N. Lu, and Y.-L. Lo, "Evaluation of AMI and SCADA data synergy for distribution feeder modeling," IEEE Transactions on Smart Grid, vol. 6, no. 4, pp. 1639-1647, 2015.

[23] R. Olfati-Saber, "Distributed Kalman filtering for sensor networks," in Proc. of the Conference on Decision and Control, 2007, pp. 5492-5498.

[24] _ , "Distributed Kalman filter with embedded consensus filters," in Proc. of the Conference on Decision and Control, 2005, pp. 8179-8184.

[25] F. Cattivelli and A. H. Sayed, "Diffusion distributed Kalman filtering with adaptive weights," in Proc. of the Conference Record of the Forty Third Asilomar Conference on Signals, Systems and Computers, 2009, pp. 908-912.

[26] F. S. Cattivelli and A. H. Sayed, "Diffusion strategies for distributed Kalman filtering and smoothing," IEEE Transactions on Automatic Control, vol. 55, no. 9, pp. 2069-2084, 2010.

[27] M. Malinowski, A. Milczarek, R. Kot, Z. Goryca, and J. T. Szuster, “Optimized energy-conversion systems for small wind turbines: Renewable energy sources in modern distributed power generation systems," IEEE Power Electronics Magazine, vol. 2, no. 3, pp. 16-30, 2015.

[28] R. M. Imran, D. Akbar Hussain, and Z. Chen, "LQG controller design for pitch regulated variable speed wind turbine," in Proc. of the International Energy Conference. IEEE, 2014, pp. 101-105.

[29] C. Jauch and S. Hippel, "Hydraulic-pneumatic flywheel system in a wind turbine rotor for inertia control," IET Renewable Power Generation, vol. 10, no. 1, pp. 33-41, 2016.

[30] A. D. Wright, Modern control design for flexible wind turbines. National Renewable Energy Laboratory Golden, CO, USA, 2004.

[31] Y. Liu and D.-L. Yu, "Robust fault detection for wind turbine systems," in Proc. of the International Conference on Automation and Computing, 2014, pp. 38-42. 
[32] H. A. Mohammadpour, Y.-J. Shin, and E. Santi, "SSR analysis of a DFIG-based wind farm interfaced with a gate-controlled series capacitor," in Proc. of the Applied Power Electronics Conference and Exposition, 2014, pp. 3110-3117.

[33] Z. Wu, W. Gao, D. Yang, and Y. Shi, "Comprehensive modeling and analysis of permanent magnet synchronous generator-wind turbine system with enhanced low voltage ride through capability," in Proc. of the Energy Conversion Congress and Exposition, 2012, pp. 2091-2098.

[34] X. Yu, J. W. Modestino, and X. Tian, "The accuracy of Markov chain models in predicting packet-loss statistics for a single multiplexer,' IEEE Transactions on Information Theory, vol. 54, no. 1, pp. 489-501, 2008

[35] P. Almstrom, M. Rabi, and M. Johansson, "Networked state estimation over a Gilbert-Elliot type channel," in Proc. of the Joint 48th IEEE Conference on Decision and Control and 28th Chinese Control Conference, 2009, pp. 2711-2716.

[36] T. Jiang, I. Matei, and J. Baras, "A trust based distributed Kalman filtering approach for mode estimation in power systems," in Proc. of the First Workshop on Secure Control Systems, 2010.

[37] E. Aranda-Escolastico, M. Guinaldo, and S. Dormido, "Stability of output event-based control systems through quadratic trigger functions," in Proc. of the International Conference on Emerging Technologies and Factory Automation, 2015, pp. 1-7.

[38] R. D. Zimmerman, C. E. Murillo-Sánchez, and R. J. Thomas, "Matpower: Steady-state operations, planning, and analysis tools for power systems research and education," IEEE Transactions on Power Systems, vol. 26, no. 1, pp. 12-19, 2011.

[39] J. Löfberg, "YALMIP: A toolbox for modeling and optimization in Matlab," in Proc. of the International Symposium on Computer Aided Control Systems Design, 2004, pp. 284-289.

[40] Y. Wang, P. Yemula, and A. Bose, "Decentralized communication and control systems for power system operation," IEEE Transactions on Smart Grid, vol. 6, no. 2, pp. 885-893, 2015.

[41] A. J. Wood and B. F. Wollenberg, Power generation, operation, and control. John Wiley and Sons, 2012.

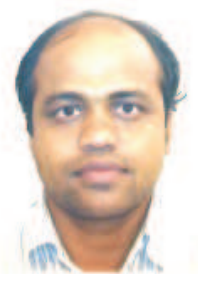

and smart grid.

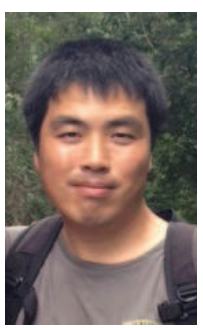

Li Li received Ph.D degree from the University of California, USA. He is servicing as a senior lecturer in school of Electrical, Mechanical and Mechatronic Systems at University of Technology Sydney, Australia. His current research interests are robust control systems, distributed model predictive control of power systems, model reduction of power systems, control on microgrids, vehicle-to-grid coordination method and
Electrical, Mechanical and Mechatronic Systems at University of Technology Sydney, Australia. His research interests are in the theoretical and algorithmic in signal processing and infimizations, statistical learning and distributed optimizations and adaptive algorithms, as well as their applications in communications, networked systems smart grid market.

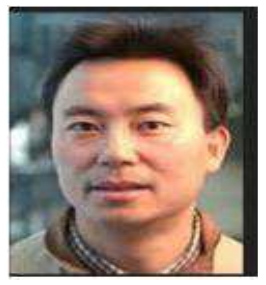

Steven Su received his $\mathrm{PhD}$ in Control Engineering from the Australian National University in May 2003. He was then a research fellow for nearly four years in the Faculty of Engineering at the University of NSW. His main research interests are biomedical instrumentation, physiological variable modeling, data acquisition and distribution, process control and system identification. He is currently an Associate Professor in the School of EMMS, and a core member of the Center for Health Technologies at UTS. 